ESJ Social Sciences

\title{
Représentations sociales et usages des jardins publics dans la commune de Treichville (Côte d'Ivoire)
}

\author{
Gueu Noël Igbou \\ Doctorant en sociologie, \\ Université Félix Houphouët Boigny (Abidjan-Côte d'Ivoire)
}

Doi:10.19044/esj.2021.v17n27p149

Submitted: 15 June 2021

Accepted: 10 July 2021

Published: 31 August 2021
Copyright 2021 Author(s)

Under Creative Commons BY-NC-ND

4.0 OPEN ACCESS

Cite As:

Igbou G.N. (2021). Représentations sociales et usages des jardins publics dans la commune de Treichville (Côte d'Ivoire). European Scientific Journal, ESJ, 17 (27), 149.

https://doi.org/10.19044/esj.2021.v17n27p149

\section{Résumé}

Les jardins publics sont des espaces verts urbains qui ont pour vocation l'agrément et le loisir des citadins. Ces espaces assurent pleinement ces fonctions lorsque les usages dont ils font l'objet par les populations sont conformes à ceux assignés par les urbanistes. Dans l'agglomération abidjanaise en général, et particulièrement dans la commune de Treichville, les usages premiers auxquels les jardins publics étaient destinés sont détournés à d'autres fins par les populations. Les jardins existants font l'objet d'occupation anarchique. L'objectif de cette étude est d'analyser les pratiques liées aux représentations sociales que les populations ont des jardins publics à Treichville. Ces pratiques sont perceptibles à travers les usages de ces espaces verts. La démarche méthodologique a consisté à l'observation directe des jardins publics et à l'entretien semi-directif. Les résultats révèlent que les jardins font l'objet d'usage spontané et illégal par les populations. Ces espaces sont transformés d'une part en lieux de pratique d'activités lucratives, de dépotoir d'ordure et d'aisances, de séchage de linges et d'aliments. D'autre part, ils sont transformés en lieux de parcours d'animaux, d'habitation, de refuge des bandits et en terrains de jeux. Ces usages entrainent la dégradation de ces équipements urbains. L'éducation à l'environnement de la population, une importance accordée aux jardins publics dans toute politique environnementale et d'aménagement du cadre de vie des pouvoirs publics permettraient la préservation de ces infrastructures. 
Mots clés : Jardins publics, représentations sociales, usages, Treichville, Côte d'Ivoire

\title{
Social Representations and Uses of Public Gardens in the Township of Treichville, Ivory Coast
}

\author{
Gueu Nö̈l Igbou \\ Doctorant en sociologie, \\ Université Félix Houphouët Boigny (Abidjan-Côte d'Ivoire)
}

\begin{abstract}
Public gardens are urban green spaces intended for the enjoyment and leisure of city dwellers. These spaces fully perform these functions when the uses to which they are subject by the populations comply with those assigned by town planners. In the Abidjan agglomeration in general, and particularly in the township of Treichville, the primary uses for which the public gardens were intended are being diverted to other ends by the population. The existing gardens are subject to anarchic occupation. The objective of this study is to analyse the practices linked to the social representations that the populations have of public gardens in Treichville. The practices are perceptible through the uses of these green spaces. The methodological approach consisted of direct observation of public gardens and semi-structured interview. The results reveal that the gardens are subject to spontaneous and illegal use by the populations. On the one hand, these spaces are transformed into places for practicing lucrative activities, dumping ground for garbage and toilets, drying clothes and food. On the other hand, they are transformed into places where animals can roam, dwellings, refuge for bandits and playgrounds. These uses lead to the degradation of these urban amenities. Environmental education of the population, an importance given to public gardens in any environmental policy and the development of the living environment of the public authorities would allow the preservation of these infrastructures.
\end{abstract}

Keywords: Public gardens, social representations, uses, Treichville, Ivory Coast

\section{Introduction}

L'urbanisation est un phénomène global qui se manifeste par une augmentation continue de la population des zones urbaines. Ce phénomène qui se caractérise par la croissance des villes et de leur périphérie, a des effets néfastes sur l'environnement urbain. Ce sont entre autres la pollution de l'air, 
de l'eau, des sols, la dégradation de l'environnement et du cadre de vie, la disparition du couvert végétal.

Face à la problématique de la dégradation de l'environnement urbain, les espaces verts apparaissent comme indispensable dans la ville. La reconstitution, la conservation et la mise en valeur des espaces naturels (Bonhomme, 2012) sont nécessaires pour le développement durable de la ville. La ville a donc un devoir de protection, d'amélioration et de valorisation (Rolland, 2009) de ces équipements urbains.

Ces espaces naturels remplissent de multiples fonctions importantes qui peuvent se répartir sous les trois piliers du développement durable: écologique, social, économique. Au niveau écologique, les espaces verts participent à la protection de l'environnement et à l'équilibre naturel (biodiversité, régulation thermique, qualité de l'air, écoulement des eaux et protection des sols) (Laille et al.,2013). Concernant le volet social, les espaces verts améliorent le bien-être physique et psychologique, développent des aspects sociaux différents à l'intérieur du tissu urbain. Ils constituent également un outil pédagogique, un lieu de découverte et d'observation de la nature. Sur le plan économique, les espaces verts génèrent de l'emploi, augmentent la valeur foncière des propriétés et l'attraction des villes. Ils constituent un atout économique non négligeable pour les municipalités (Lessard et al., 2008).

La norme internationale en matière des espaces verts recommandée par l'Organisation Mondiale de la Santé (OMS) est de $10 \mathrm{~m}^{2}$ par habitant (Polorigni et al., 2015). Ces espaces sont multiformes et variés. Ce sont entre autres les espaces verts d'accompagnement des constructions, les parcs urbains, les squares et les jardins publics. Cette typologie n'est pas exhaustive.

Le jardin public qui est le centre d'intérêt de cette étude se définit comme « un espace vert urbain, enclos, à dominante végétale, protégé des circulations générales, libres d'accès, conçu comme équipement public et géré comme tel » (Merlin et Choay, 1988). Ces espaces ont pour vocation l'agrément et le loisir des citadins. La conservation de ces équipements urbains nécessite un suivi de ceux-ci par les pouvoirs publics d'une part, et d'autre part un usage par la population conforme aux fonctions assignées à ces espaces par les urbanistes.

Dans l'agglomération abidjanaise, les espaces verts en général et en particulier les jardins publics subissent de nombreuses pressions et ne bénéficient pas toujours de protection ni de gestion adéquate (De Villers et Machteld, 2006). La commune de Treichville n'échappe pas à cette situation du recul de ces espaces verts. Bon nombre de jardins publics ont disparu pour cause de morcellement, d'occupations anarchiques, de construction de bâtiments administratifs (Tra et Igbou, 2017). C'est le cas du jardin public du quartier Yobou Lambert (ex Biafra) dont le site abrite le Centre de Recherche 
en Ecologie, le Centre d'Education Communautaire pour l'Enfance Amicha François, l'Institut d'Histoire et d'Archéologie, le Commissariat du 29ème arrondissement (Kouamé et Kouamé, 2012). De même, l'immeuble Treich Center aux avenues 1 et 2, et la place Aboussouan sont en lieu et place des jardins publics disparus (Kouassi et Amani, 2017).

La situation actuelle des jardins publics existants dans la commune de Treichville est préoccupante. En effet, ces espaces manquant d'entretien, sont dans un état d'abandon et de dégradation avancée. De plus, l'usage de ces équipements urbains est contraire à celui assigné par les concepteurs, c'est-àdire des lieux de repos, d'agrément. Ces jardins font l'objet d'occupation anarchique par les populations. Ces espaces sont transformés par les populations en lieux de pratique d'activités lucratives, lieux d'aisances et de dépotoir d'ordure. En outre, ces jardins sont transformés en lieux de séchage de linge et d'aliment, lieux de parcours d'animaux, lieux d'habitation, lieux de refuge des bandits et en terrains de jeux.

Ces usages sont des pratiques guidées par les représentations sociales des populations vis-à-vis de ces espaces. Partant de là, comment les représentations sociales construisent-elles les usages des jardins publics dans la commune de Treichville? L'objectif de ce travail est d'analyser les pratiques liées aux représentations sociales que les populations ont des jardins publics. L'hypothèse de cette étude est la suivante : l'usage des jardins publics dans la commune de Treichville est déterminé par la représentation sociale que les populations se font de ceux-ci.

\section{1- Matériel et méthodes}

\section{1- Présentation de la zone d'étude}

La présente étude a été menée à Treichville, une commune d'Abidjan. La commune de Treichville ( $5^{\circ} 18^{\prime} 12^{\prime \prime} \mathrm{N}$ et $\left.4^{\circ} 00^{\prime} 33^{\prime} ' \mathrm{~W}\right)$ est située dans la partie sud de la ville d'Abidjan sur l'île de petit Bassam au milieu de la lagune Ebrié. Elle est délimitée à l'est par la commune de Marcory ; et partage des limites en pleine lagune avec la commune du Plateau au nord, celle de Port Bouët au sud et celle de Yopougon à l'ouest (voir Figure 1). La commune de Treichville a un relief plat et bénéficie d'un climat de type subéquatorial humide et chaud. La température moyenne annuelle est de $25,9^{\circ} \mathrm{C}$ et les précipitations sont en moyenne de $1441 \mathrm{~mm}$ par an (www.climate-data.org).

La commune de Treichville compte 43 quartiers, et s'étend sur une superficie de $9 \mathrm{~km}^{2}$. La population de Treichville est estimée à 102580 habitant dont 51254 Hommes et 51326 Femmes (RGPH, 2014). 


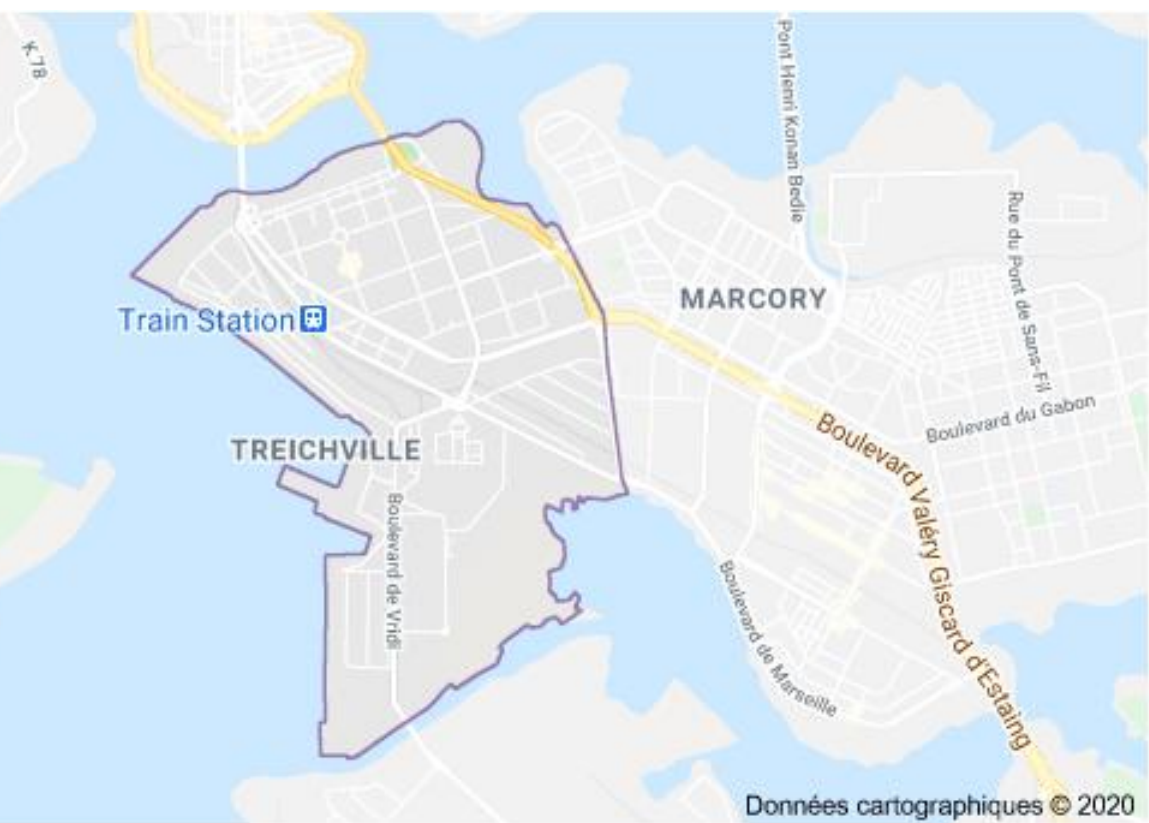

Figure 1 : Localisation de la commune de Treichville

Source : Google Maps, 2020

\section{2- Matériel et population d'étude}

Le matériel de la présente étude est représenté par les six (6) jardins publics de la commune de Treichville. Ce sont le jardin canal aux bois ou jardin public quartier habitat Craonne, le jardin Paul Godeau, le jardin public CNPS, le jardin Statue, le jardin bourse du travail et le jardin public cité douane.

La population cible de cette étude est constituée de quatre catégories d'acteurs. Les usagers des jardins publics, les habitants riverains de ces espaces, les responsables des quartiers à proximité des jardins publics (les Présidents de comité de gestion du quartier (CGQ)) constituent les trois premières catégories d'acteurs. L'étude prend également en compte les pouvoirs publics en charge de la gestion des jardins publics (les agents municipaux de la commune de Treichville et les agents de la Direction des Parcs et Jardin du District Automne d'Abidjan) qui sont la quatrième catégorie d'acteurs.

\section{3- Collecte des données}

Pendant la phase de collecte des données, les six (6) jardins publics de la commune de Treichville ont été visités.

Dans cette étude, la démarche qualitative du point de vue de l'approche analytique a été privilégiée. Les techniques de collecte des données utilisées sont la recherche documentaire, l'observation directe et l'entretien semi- 
directif. Concernant la recherche documentaire, les écrits qui ont été pris en compte sont ceux de la problématique générale de la gestion, de l'occupation et de la dégradation de l'espace vert en milieu urbain. Quant à l'observation, elle a porté sur l'état de conservation et de dégradation des jardins publics d'une part, et d'autre part sur les pratiques en cours dans ces espaces. En ce qui concerne l'entretien, elle s'articule principalement autour de l'utilisation de ces espaces par les usagers.

L'échantillon à choix raisonné est le type d'échantillonnage privilégié. Il est constitué de quatre (4) présidents de comité de gestion du quartier, vingtcinq (25) usagers des jardins publics, dix (10) riverains de ces espaces, trois (3) agents du District Autonome d'Abidjan et cinq (5) agents municipaux de la commune de Treichville. Ainsi, la taille de l'échantillon est de quarantesept (47) enquêtés.

\section{4- Méthodes de traitement et d'analyse des données}

Le dépouillement des données recueillies a été fait de façon manuelle. La technique d'analyse des données utilisée est l'analyse qualitative de contenu qui s'intéresse au fait que des thèmes, des mots ou des concepts soient ou non dans un contenu. Celle privilégiée est l'analyse thématique transversale. L'analyse thématique sert à dégager les thèmes présents dans un corpus pour ensuite les analyser. Quant au traitement des données, le traitement sémantique a été privilégié. Il consiste à l'analyse empirique des idées, des mots et de leur signification. Le type de traitement sémantique utilisé est l'analyse lexicale qui consiste à étudier les mots clés utilisés par les enquêtés.

\section{2- $\quad$ Résultats}

Les jardins publics occupent une place importante en milieu urbain. Ainsi, des pratiques y sont développées et les usagers établissent des relations particulières avec ces espaces. Chaque individu construit à l'égard de ces espaces des représentations sociales. Les représentations sociales des populations vis-à-vis de ces équipements correspondent donc aux valeurs qu'elles accordent à ceux-ci, à la façon dont elles les vivent au quotidien, les jugent et les sentent.

A Treichville, les populations se représentent les jardins publics comme des lieux de pratique d'activités lucratives, des réserves foncières, des terrains de jeux. Les pratiques liées à ces représentations sociales sont perceptibles à travers les usages que les populations font des jardins publics. Ceux-ci concourent à la dégradation de ces espaces. Les jardins font l'objet d'occupation anarchique par les populations et les usages de ces espaces varient d'un groupe d'individus à un autre selon leurs intérêts. Cette partie du travail consiste à monter ces usages. 


\section{1- Les jardins publics : lieux de pratique d'activités lucratives}

Les jardins publics dans la commune de Treichville sont pris d'assaut par la population dans le but d'exercer des activités lucratives. Les comportements de celle-ci sont liés à la pauvreté et au chômage grandissants. En effet, les crises (crise économique, la crise politico-militaire de 2002) que la Côte d'Ivoire a connues, ont aggravé la situation de la pauvreté et du chômage. Le taux de pauvreté en Côte d'Ivoire est estimé à 46,3\%, et la ville d'Abidjan abrite 9,3\% du taux national (INS, ENV 2015). Quant au chômage, Abidjan connaît un taux de chômage de 13,4\% (INS, ENV 2015), largement supérieur au taux national qui est de 9,4\% (ONP, 2015). La jeunesse est la frange de la population la plus touchée par le chômage. En Côte d'ivoire, les jeunes représentent $63,0 \%$ de la population en âge de travailler, et résident majoritairement en milieu urbain avec un taux de 52,8\% dont $21,3 \%$ dans la ville d'Abidjan et 31,5\% dans les autres villes (INS, ENSESI 2016).

Face à cette situation de pauvreté et chômage grandissants, la population n'a autre alternative que de chercher refuge dans l'informel pour survivre. Pour exercer leurs activités, celle-ci occupe les espaces publics (les rues, les trottoirs, les espaces verts, ...) où elle échappe parfois au contrôle des agents municipaux. Ainsi, les jardins publics sont envahis par les activités du secteur informel. C'est le cas du jardin statue devenu un lieu où se développent les activités informelles (Photo 1).

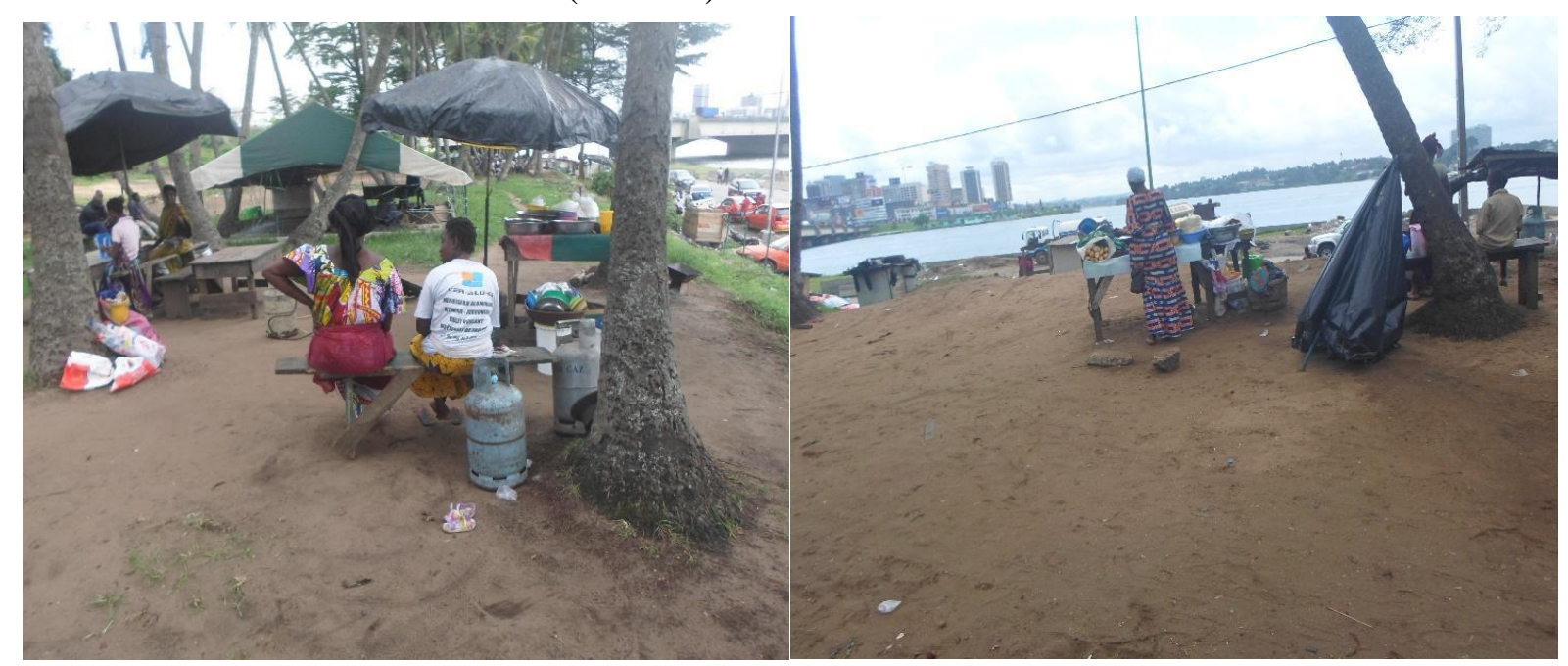

Photo 1 : Les vendeuses installées dans le jardin Statue Source : Cliché auteur, avril 2017

En plus d'être des lieux de pratique d'activités lucratives, les jardins deviennent parfois des lieux de dépotoir d'ordure et d'aisances. 


\section{2- Les jardins publics, lieux de dépotoir d'ordure et d'aisances}

Les usagers et les riverains des jardins publics ont des comportements peu respectueux de l'environnement qui se traduisent par la transformation de ces espaces d'une part en lieux de dépotoir d'ordure, et d'autre part en lieux d'aisances.

S'agissant de l'utilisation de ces espaces comme des dépotoirs, elle est l'œuvre d'une part des usagers et d'autre part des riverains. En effet, certains usagers laissent beaucoup d'ordures après leur passage dans les jardins. L'on y voit entre autres les emballages en plastique, les bouts de papier, les sachets trainés partout dans ces lieux. Ces espaces deviennent ainsi des lieux insalubres. Les propos d'un enquêté montrent l'état insalubre de cet espace. Il dit ceci : «ce jardin-là vraiment, c'est très sale. Pas d'entretien, manque d'entretien, et puis bon, les gens jettent les ordures n'importe comment». Selon les propos de cet enquêté, le jardin est insalubre du fait qu'il manque d'entretien d'une part, et d'autre part du comportement des usagers non respectueux de l'environnement.

A la question de savoir qui est responsable de l'état insalubre du jardin ?, un usager tient les propos suivants : «c'est nous, c'est toujours nous. C'est ceux qui viennent fréquenter le jardin public ». Un autre usager va dans le même sens que le précédent. Il affirme ceci : «ce sont ceux qui y sont quotidiennement, les occupants qui salissent le jardin. Je vois des personnes qui viennent manger, qui font de cet espace un dépotoir. Je suis là, ça vaut une heure de temps. J'ai assisté à plusieurs personnes qui sont venues manger, qui ont fini de manger, qui ont déposé heur les restes, et qui sont parties. Donc, pour elles, ce n'est pas un coin reposant, mais un dépotoir ». Il ressort des propos de cet enquêté que les principaux responsables de l'état insalubre des jardins sont les usagers. Ceux-ci ne prennent pas soin de garder ces lieux salubres, ils laissent les déchets après leur passage.

En outre, ces lieux sont pour certains riverains une aubaine pour se débarrasser des ordures ménagères, vu leur caractère public. Ainsi, les dépôts sauvages d'ordure sont récurrents dans les jardins. C'est ce qui ressort des propos d'un agent municipal de la commune de Treichville. A la question de savoir : quelle est l'attitude de la population face aux jardins publics ?, il dit ceci : "Ce qu'on constate plus c'est le déversement des ordures dans les espaces verts ». Selon les dires de cet agent, les jardins sont transformés par la population en dépotoir d'ordure. En demandant son avis sur le comportement de la population, il affirme ceci : «le comportement n'est pas ça. Elle n'est pas éduquée à cela. Ce sont les comportements qui ne sont pas éco citoyens, qui nuisent fortement au bon fonctionnement de ces espaces ». Il ressort de ces propos que les comportements de la population impactent négativement ces espaces et conduisent à leur dégradation. 
La présence des dépôts sauvages d'ordure dans les jardins Paul Godeau et Statue met en lumière cette situation (photo 2).

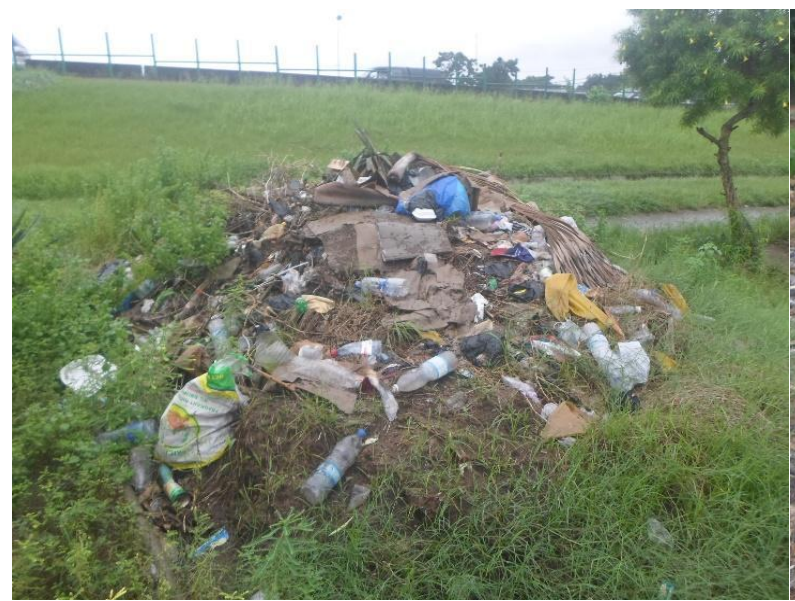

A : Jardin Paul Godeau

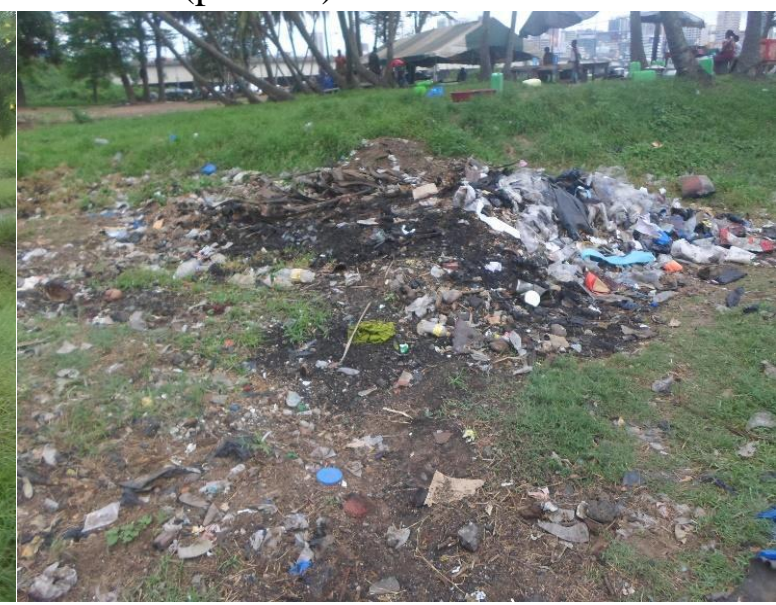

B : Jardin Statue

Photo 2 : Présence des tas d'ordures dans les jardins publics Source : Cliché auteur, avril 2017

Concernant l'utilisation des jardins en tant que lieux d'aisances ; ces espaces sont pour certains usagers des urinoirs et des WC publics. Ces pratiques rendent ces lieux insalubres. C'est ce qui ressort des propos d'un enquêté. Il déclare ceci : "le jardin est très sale. Puis, les gens pissent partout. On ne sait pas que c'est un jardin public. Un jardin public où les gens peuvent venir se reposer à l'aise, ils font du n'importe quoi ». Un usager va dans le même sens que cet enquêté. Il soutient que les principaux responsables de l'état insalubre des jardins publics sont les usagers. Ces propos sont les suivants : «ce sont les citoyens, ceux qui fréquentent le jardin. Parce que heur, on ne peut pas dire que ce sont les autorités. C'est nous-mêmes en fait qui salissons, qui pissons un peu partout, qui déféquons un peu partout. Bon! c'est nous même». Face à ces pratiques non respectueuses de l'environnement, un président du comité de gestion de quartier dans la commune de Treichville exprime son étonnement. Il dit ceci : « il y a des fois tu arrives dans le jardin, hein, quelqu'un est venu déféquer sur le banc. C'est impensable quoi! ».

Ces espaces sont aussi des lieux de séchage des linges et d'aliment.

\section{3- Les jardins publics : lieux de séchage de linges et d'aliments}

Les jardins publics dans la commune de Treichville sont parfois affectés à d'autres usages par la population. Ils font l'objet de forte pression par les riverains qui les utilisent pour le séchage de linges.

En plus d'être des lieux de séchage de linges, les jardins publics sont également utilisés par les riverains comme lieux de séchage d'aliment. Les 
pratiques en cours dans ces espaces attirent l'attention des visiteurs de ces endroits. A ce sujet un usager pointe du doigt la responsabilité les pouvoirs publics pour leur inaction face à ces pratiques. Il dit ceci : «voilà, ils sont entrain de sécher attiéké. Bon! tout ça là, je peux dire bon!, peux être que la mairie même est dedans quoi! Parce que il n'y a pas un bon suivi des jardins ». Pour cet enquêté, le manque de suivi de ces espaces et l'inaction des pouvoirs publics face à l'occupation des jardins favorisent ces pratiques. C'est le cas du jardins public Statue transformé en lieu de séchage de linges et d'aliments.

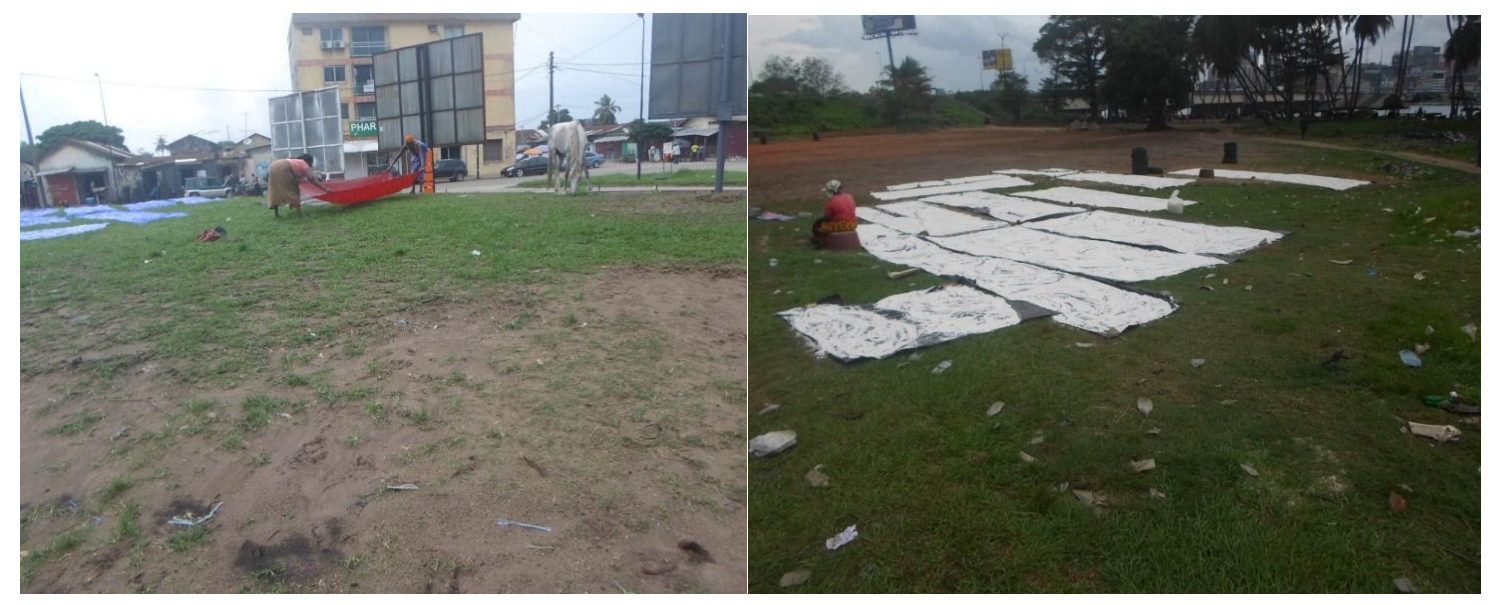

Photo 3 : Le jardin Statue transformé en lieu de séchage de linges et d'aliments Source : Cliché auteur, avril 2017

Les jardins publics sont également des lieux de parcours d'animaux.

\section{4- Les jardins publics, lieux de parcours d'animaux}

Certains jardins servent de lieux de parcours d'animaux. C'est le cas des jardins statue et bourse du travail utilisés par les riverains pour faire paître des chevaux et des moutons.

La transformation de ces espaces en lieux de parcours des animaux a un impact négatif sur ces sites. En effet, la présence des animaux dans les jardins détruit les espèces florales et conduit à la dégradation de ces espaces. Le comportement de certains riverains s'explique par le fait que les habitants de la commune de Treichville n'appréhendent pas les espaces verts de la même manière. Certains connaissent l'utilité de ces espaces. Par contre, d'autres ignorent totalement leur importance. C'est cette catégorie de la population qui fait paître les animaux dans les jardins publics destinés pour le repos des citadins. Ces mauvaises pratiques conduisent inéluctablement à la dégradation de ces espaces.

Ce comportement non respectueux de l'environnement de la population s'explique aussi par le laisser-faire des pouvoirs publics face aux 
pratiques de celle-ci. En effet, les pouvoirs publics ont pour rôle de sensibiliser les habitants sur l'usage approprié des jardins publics. Cependant, ces espaces deviennent des lieux de parcours d'animaux sans que les autorités n'interviennent. Cela amène à s'interroger si les espaces verts en général et particulièrement les jardins publics font partie des priorités des pouvoirs publics dans la politique environnementale et d'aménagement du cadre de vie. Ce manque d'intérêt pour ces espaces se traduit par l'abandon de ces sites qui ne sont pas entretenus. Celui-ci est l'une des causes de la dégradation avancée de ces équipements urbains.

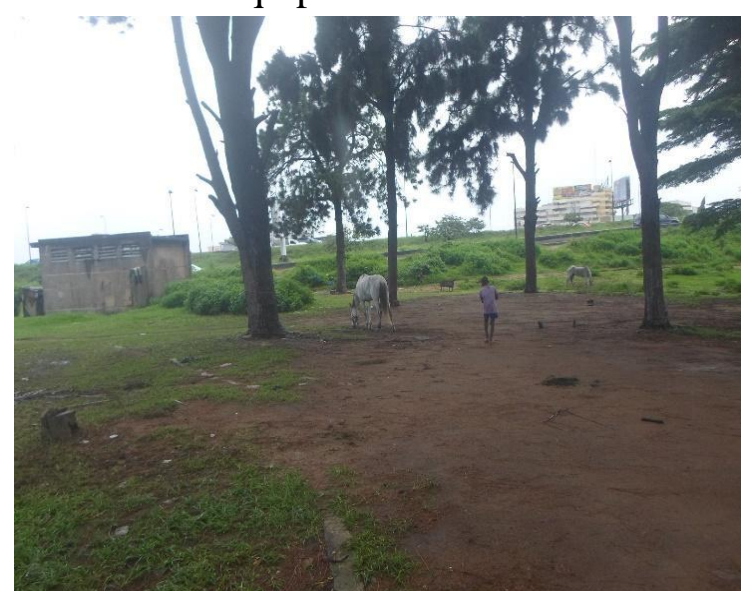

A : Jardin Statue

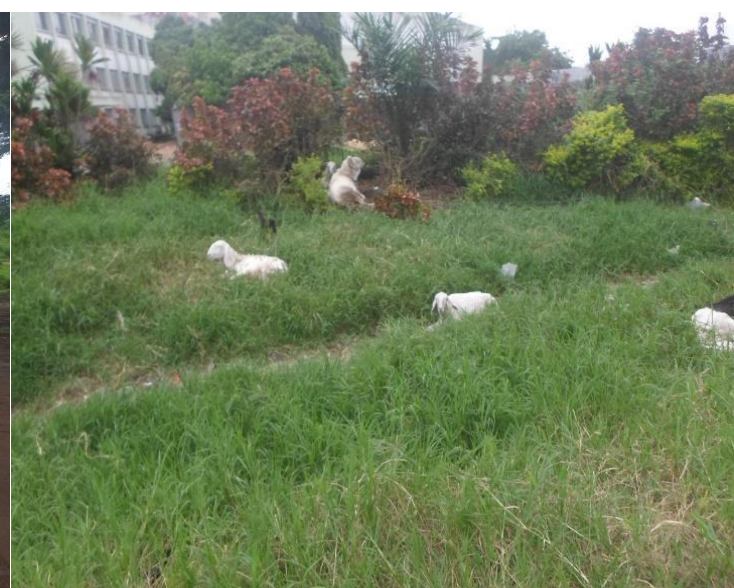

B : Jardin bourse du travail

Photo 4 : Des jardins transformés en lieux de parcours d'animaux Source : Cliché auteur, avril 2017

Outre des lieux de parcours d'animaux, ces espaces sont des lieux d'habitation.

\section{5- Les jardins publics : lieux d'habitation}

Les jardins publics font l'objet d'occupation par des individus qui les utilisent comme dortoir et habitation. Cette occupation est temporelle ou permanente selon le cas.

S'agissant de l'occupation temporelle, elle est l'œuvre de ceux qui exploitent ces lieux comme dortoir. A titre d'exemple, le jardin public CNPS et le jardin Paul Godeau servent de dortoir pour les sans- abris. 


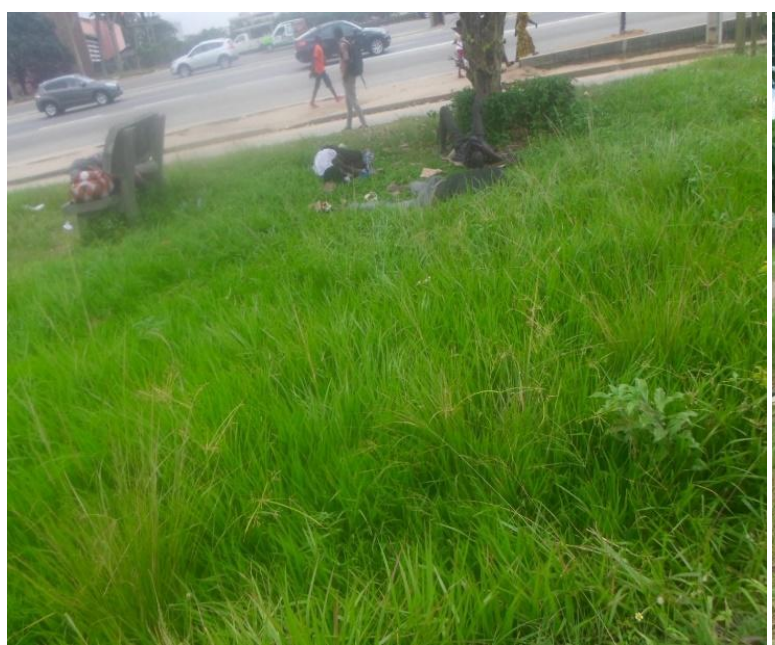

A : Jardin public CNPS

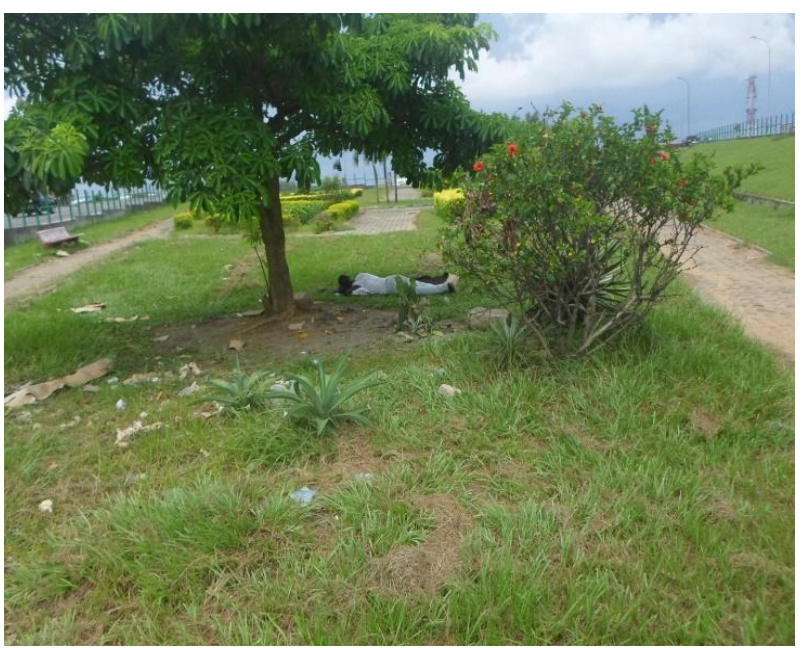

B : Jardin Paul Godeau

Photo 5 : Des jardins transformés en dortoirs

Source : Cliché auteur, avril 2017

Quant à l'occupation permanente, elle se traduit par la construction des habitats spontanés dans les jardins. C'est le cas du jardin Paul Godeau et celui de la bourse du travail où l'on note la présence d'habitat spontané.

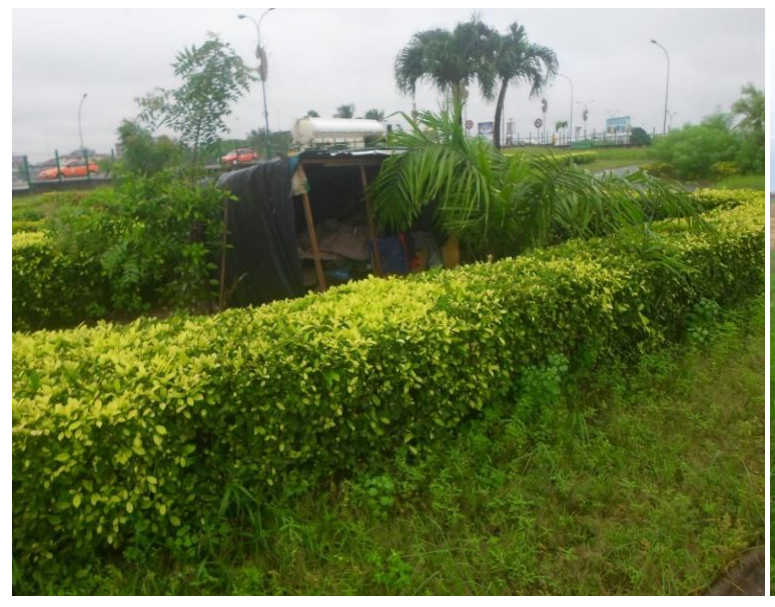

A : Jardin Paul Godeau

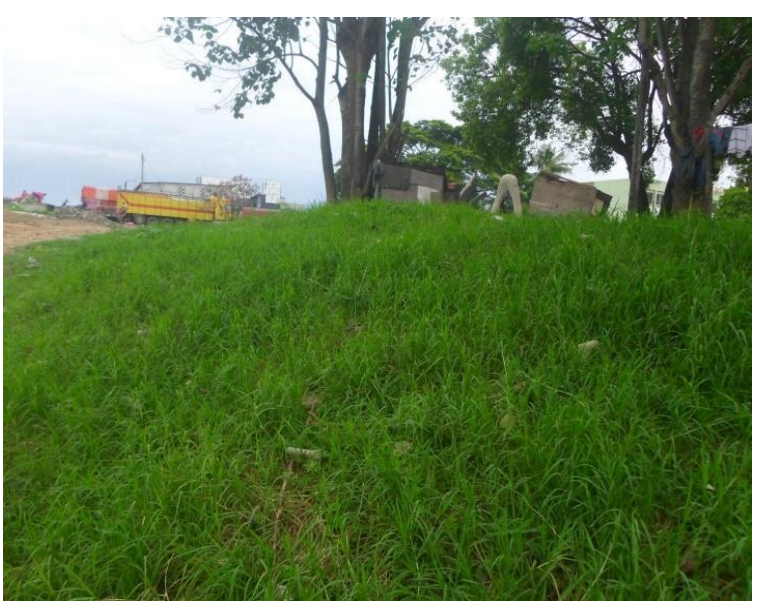

B : Jardin bourse du travail

Photo 6 : Présence d'habitat spontané dans les jardins publics

Source : Cliché auteur, avril 2017

La catégorie d'individus utilisant les jardins publics comme dortoirs et lieux d'habitation est composée essentiellement de marginaux. Ce sont entre autres les malades mentaux (fous), les enfants de la rue, les toxicomanes, les délinquants. A ce sujet, un président de comité de gestion du quartier s'exprime en ces termes : «Bon! le jardin est bien situé, bien menu. Mais, 
heur. Souvent, heur, des vrais propriétaires, ce sont nos frères heur malades. Hein, soit bon, des fous. Hein, bon heur des, je ne sais pas hein! Heur, ça fait aussi d'autres viennent se délasser pour fumer quoique ce soit. Mais, voilà un peu le regret que nous avons hein. Sinon, heur c'était bien venu».

L'utilisation de ces espaces comme lieux d'habitation n'est pas le seul type d'usage en cours sur ces sites. Ils sont également des terrains de jeu.

\section{6- Les jardins publics, terrains de jeux}

Les jardins publics dans la commune de Treichville sont constamment affectés à d'autres usages autre que ceux auxquels ils sont destinés, c'est-àdire lieu de repos, de loisir. Certains jardins sont utilisés comme des terrains de jeu. A titre d'exemple, l'on peut citer le jardin Statue qui en son sein, un terrain a été créé par les riverains, et sert de lieu d'entrainement pour les jeunes.

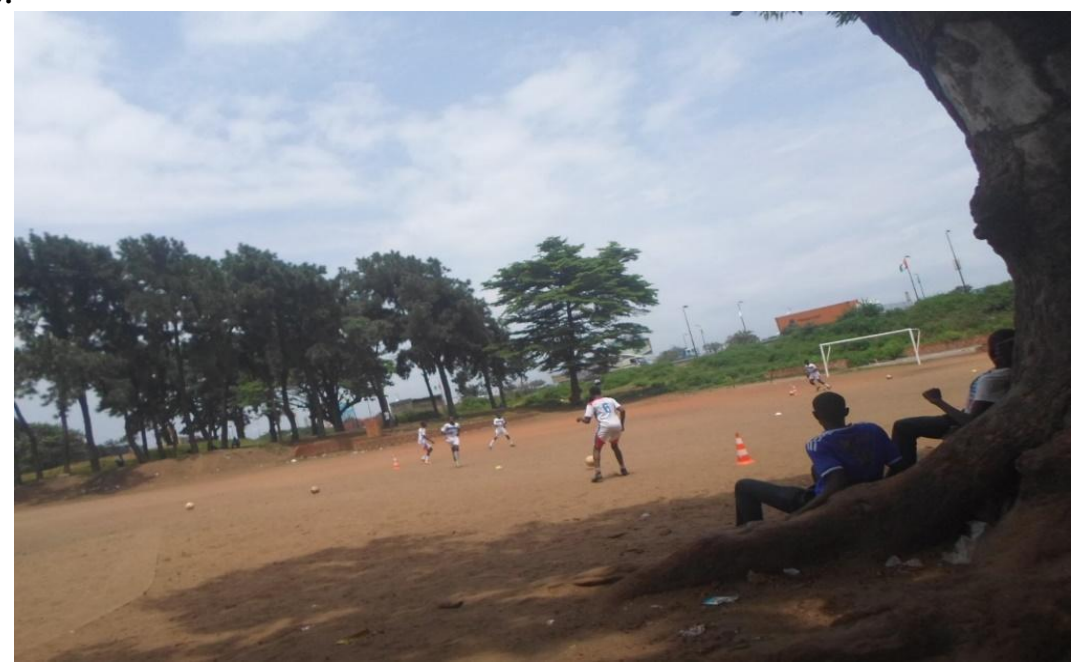

Photo 7: Le jardin statue transformé en terrain de jeux Source : Cliché auteur, avril 2017

L'absence de terrains de jeu dans certains quartiers est à l'origine de ce phénomène d'occupation des jardins. C'est ce qui ressort des propos du président du comité de gestion de quartier (CGQ) du quartier Yobou Lambert de Treichville. Il dit ceci : "l'autre jardin qui est à la montée du pont; puisqu'ils ont fermé notre complexe. Bon!, on était obligé de donner ça aux enfants pour qu'ils s'entrainent là-bas. Pour le moment, c'est notre terrain hein. Notre complexe est fermé. Actuellement la jeunesse s'entraine là-bas». Selon le responsable de ce quartier, c'est la fermeture de leur infrastructure sportive qui amène la jeunesse à utiliser ce jardin comme terrain de jeu. A la question de savoir, qu'est-ce qu'il faut faire pour la pérennité de ce jardin ?, il affirme ceci : «Bon!, si le district d'Abidjan prend ses responsabilités, il donne des aires de jeux aux jeunes gens. Si le complexe est ouvert, on va les 
aider à bien arranger leur jardin. On va leur dire, ne venez plus ici. C'est parce que il n'y a pas d'espace de jeux que les enfants sont obligés d'aller dans le jardin ». Pour lui, la réouverture du complexe sportif du quartier va mettre fin à l'exploitation de cet espace comme terrain de jeu. Il est bon de noter que l'absence des équipements sportifs est l'une des causes de la destruction des jardins publics.

Les jardins publics sont des équipements urbains qui participent à l'amélioration du cadre de vie des citadins. Toutefois, ces espaces sont une source d'inquiétude pour la population quand ils deviennent des repères des bandits.

\section{7- $\quad$ Les jardins publics : lieux de refuge des bandits}

Les jardins publics sont des espaces publics dont l'accès est permis à toutes les couches sociales. Ces espaces se transforment parfois en des repères d'habitations des délinquants. Ainsi, ces lieux destinés à l'agrément du public, deviennent une source d'insécurité et troublent la quiétude des populations. Certains usagers sont par moments agressés. Ces agressions récurrentes empêchent la population à fréquenter les jardins publics. C'est ce qui ressort des propos du président de CGQ du quartier Anatole France. Il dit ceci : «Au niveau des jardins publics, il faut dire que, comme nous-mêmes, on a un jardin public ici. Hein, et à cause des agressions, hein, du côté de la caisse, on interdit même nos enfants et à certaines personnes d'elles-mêmes, heur, d'aller s'assoir là-bas hein. Parce que on ne sait jamais. Heur, tu es là en train de prendre de l'air, quelqu'un vient, bon il t'arrache ton portable. Ou bien, il te dépouille de tes objets. Donc c'est difficile ». Face à l'insécurité grandissante dans les jardins, les responsables de ce quartier interdisent aux habitants de fréquenter certains espaces par mesure de prévention.

Certains jardins sont également les bases arrières des délinquants. C'est à partir de ces espaces, que ceux-ci partent pour agresser les passants dans les périmètres de ces lieux. Un président de CGQ décrit ces faits en ces termes : "le deuxième jardin en montant vers le pont pour aller à Marcory, hein, souvent ils sont dans les aires là-bas. Heur, en fait c'est de tous ces coins-là que les gens, les agresseurs vont rester là-bas pour venir attaquer les gens. Ils glissent là, et puis ils te trouvent dans le mur de la caisse là pour t'agresser. Que tu sois femme ou bien garçon. En pleine journée hein. Bon, maintenant la nuit ça devient un peu plus dangereux». Il ajoute ceci : «vous voyez le mur de la caisse. Ça devient un tunnel. Donc, si tu es engagé et que tu es seul là. Les gars, ils sont assis dans le jardin. Dès que tu les as dépassés, ils te rejoignent là-bas, hein, pour t'agresser. Et puis bon, ils prennent tous tes biens et ils s'en vont ». Il ressort des propos de cet enquêté que les bandits se réunissent dans les jardins pour planifier les agressions. 
La persistance de l'insécurité dans les jardins s'explique par le manque de suivi de ces espaces par les pouvoirs et l'inaction des autorités face aux agressions. En ce qui concerne le suivi, bon nombre de ces espaces sont abandonnés et manquent d'entretien. L'état des jardins publics constitue un frein à la fréquentation de ces lieux par la population. Ces espaces ne recevant pas un grand nombre d'usagers, devient des lieux de refuge des drogués, des bandits, des délinquants ; du fait qu'ils sont délaissés. C'est ce qui ressort des propos du président du CGQ du quartier Yobou Lambert (ex Biafra) en parlant du jardin statue. Il dit ceci : «tu sais le jardin est abandonné. Quand un truc est abandonné, le banditisme s'installe. Sinon quand le jardin est délaissé comme ça là, ça devient un coin de bandit. Ils vont, ils fument la drogue làbas. Tu as vu, ça devient un coin de bandit ». Selon ce responsable du quartier, l'état d'abandon des jardins est la cause principale de l'occupation de ces espaces par les bandits, les drogués.

A propos de l'inaction des autorités, notons que celles-ci sont régulièrement informées par la population sur les cas d'agressions répétées dans les jardins. Mais, aucune action concrète de la part des autorités pour mettre fin à l'insécurité dans ces lieux. Pas même une intervention afin de dissuader les drogués, les bandits et les délinquants qui occupent les jardins. A ce sujet, voici les propos d'un agent du district: "au niveau de Paul Godeau, on dit souvent ceux qui viennent squatter le jardin, ce sont les drogués. Mais plusieurs fois les commissariats ont été informés. Mais, ils ne viennent pas. Or, il suffisait que la police descende régulièrement, c'est fini. Ils n'allaient plus venir $»$. Il ressort des propos de cet agent du district, que les autorités ne réagissent pas quand elles sont informées de la présence des drogués dans les jardins. Les dires du président de CGQ du quartier Anatole France vont dans le même sens que ceux de cet agent du district en parlant des agressions. Il dit ceci : "Bon, souvent c'est la sécurité qui nous préoccupe. Voilà, donc de temps à autre, on se rend même souvent au commissariat ou bien on avise quelques responsables de la mairie pour attirer leur attention sur le danger des agressions ». Selon les dires de cet enquêté leur souci majeur au niveau des jardins est l'insécurité. Il interpelle les autorités compétentes sur les cas d'agression dans ces espaces. Il ajoute ceci : «comme je le disait. Si hein il pouvait, bon, on ne peut pas dire que les agents en permanence doivent rester là. Mais de temps à autres, qu'ils fassent des patrouilles. Même si un véhicule de police est garé vers la caisse là, ça peut dissuader les agresseurs. Donc, s'il y avait même la police du côté de la station. Et puis bon, voilà, ça peut beaucoup dissuader les gens ». Il ressort des affirmations de ce responsable de quartier, qu'aucune action concrète n'est menée par les autorités pour dissuader les agresseurs qui opèrent en toute impunité. 


\section{3- Discussion}

L'analyse des pratiques liées aux représentations sociales que les populations ont des jardins publics révèle que les usages premiers, à savoir lieux de repos et d'agrément, auxquels ces espaces étaient destinés sont détournés à d'autres fins par la population. Ces équipements urbains sont anarchiquement occupés pour usage spontané et illégal. Cette analyse corrobore les résultats de Kouassi et al. (2017). Ils affirment que les espaces verts à Treichville sont utilisés comme des prolongement des cours familiales, des magasins pour les activités commerciales et servent des lieux de séchage de pagne artisanaux et de poudre de maïs. Ces résultats vont dans le même sens que ceux de Kassay (2010). Cet auteur soutient que les espaces verts dans la ville de Kinshasa sont occupés par les populations pour les constructions anarchiques, des marchés pirates. Ces usages conduisent inéluctablement à la dégradation de ces espaces voire leur effacement de la carte géographique.

En outre, les jardins sont transformés par la population en lieu dépôt de déchets et d'aisances. Cette attitude de la population confirme les résultats obtenus par Kouamé et al. (2012). Selon ces auteurs, les espaces verts dans la ville d'Abidjan sont choisis par la population non seulement pour se débarrasser des ordures, mais aussi pour leurs besoins naturels. Ces résultats vont dans le même sens que ceux de Nassa (2009) qui soutient que les jardins publics dans la commune de Cocody sont devenus des dépotoirs et des WC publics. Cette analyse corrobore les résultats de Kouassi et al.(2019). Ils affirment que les espaces verts dans la ville de Yamoussoukro, principalement les bois, sont des toilettes à ciel ouvert pour les populations. Ces déchets disséminés sous-tendent la pollution visuelle qui constitue sans doute une entorse à l'égard de l'environnement. Ce comportement de la population s'explique par le fait qu'elle ne donne pas trop d'importance à la préservation de l'environnement.

Ces espaces se transforment parfois en un repère des marginaux (fous, drogués, délinquants), devenant ainsi une source d'insécurité. Cette analyse corrobore les résultats de Mebirouk (2008). Cet auteur affirme que la présence permanente des sans-abris, délinquants dans les espaces verts de la ville d'Annaba sème l'insécurité et la peur dans ces endroits. Ces résultats se rapprochent de ceux de Tra et al. (2017) qui soutiennent que l'insécurité grandissante dans les espaces verts du quartier Gnanzoua dans la commune de Marcory fait que ces espaces sont perçus par la population comme des lieux d'insécurité et non des lieux de repos.

Les implantations illégales et spontanées dans les jardins publics s'expliquent d'une part par l'incapacité des pouvoirs publics de gérer et de contrôler ceux-ci et d'autre part par le manque d'éducation environnementale de la population qui ne connait pas l'importance de ces espaces naturels. Cette analyse corrobore les résultats de Tatar et al. (2016). Ils affirment que les 
espaces verts de Tébessa souffrent de l'incurie des responsables et du manque de considération des citoyens qui donnent pas trop d'importance à la préservation de l'environnement. Ces résultats se rapprochent de ceux de Polorigi et al.(2015). Selon ces auteurs, l'état des espaces verts dans la ville de Lomé met en exergue le désengagement et l'impuissance des acteurs publics chargés de la gestion de ces équipements.

\section{Conclusion}

Cette étude est une contribution à l'analyse des pratiques liées aux représentations sociales que les populations ont des jardins publics. Elle révèle que les usages auxquels les jardins étaient destinés sont détournés à d'autres fins par la population. Ces espaces font l'objet d'usage spontané et illégal par la population, les transformant en lieux de pratique d'activités lucratives, de dépotoir d'ordure et d'aisances, de séchage de linges et d'aliments, de parcours d'animaux, d'habitation, de refuge des bandits et en terrains de jeux.

Ces pratiques montrent que la représentation sociale que les populations ont vis-à-vis de ces équipements urbains est négative. Les attitudes des populations constituent une menace pour ces espaces dans la mesure où elles entrainent leur dégradation, voire leur effacement de la carte géographique.

Pour la préservation de ces infrastructures qui sont incontournables dans toute ville qui aspire à un développement durable, les pouvoirs publics doivent d'une part accorder une importance aux espaces verts en général et particulièrement aux jardins publics dans toute politique environnementale et d'aménagement du cadre de vie. D'autre part, amener les populations à avoir un comportement respectueux face aux jardins en les éduquant à l'environnement et les sensibilisant sur l'importance de ces équipements urbains.

\section{References:}

1. Bonhomme, M. (2012). Création d'un outil d'aide à la décision pour un aménagement durable des espaces verts dans les municipalités. Essai de Maîtrise, Université de Sherbrooke.

2. De Villers J., et Machteld, G. (2006). Rapport sur l'état de l'environnement bruxellois : environnement semi-naturel et espaces verts publics. Institut bruxellois pour la gestion de l'environnement (IBGE).

3. Kassay, N.-I. J. (2010). La politique publique de la gestion des espaces verts par l'hôtel de ville de Kinshasa. Afrique et Développement, $\mathrm{xxxv}$ (3)13-46.

4. Kouamé, Y. G. et Kouamé, Y. J. (2012). Evaluation de la gestion des espaces verts du District d'Abidjan en vue d'un aménagement durable. 
Mémoire de fin d'étude, Institut National Polytechnique Félix Houphouët Boigny de Yamoussoukro.

5. Kouassi R. H., Nomel G. J. R., Kouadio Y. J.-C., Ambé A. S. A. et N'guessan K. E. (2019). Perception, Attitude et Attentes des Résidents à l'égard des Espaces Verts Urbains de Yamoussoukro (Côte d'Ivoire). European Scientific Journal, 15 (3), 1857 - 7881.

6. Kouassi, B. A. A. G. et Amani, Y. C. (20017). Problématique de la gestion durable des espaces verts publics urbains en côte d'ivoire : cas de la commune de Treichville (Abidjan). Revue de Géographie Tropicale et d'Environnement, (2), 52-62.

7. Laille, P., Provendier, D., Colson, F. et Salanie, J. (2013). Les bienfaits du végétal en ville: étude des travaux scientifiques et méthode d'analyse. Plante\& Cité.

8. Lessard, G. et Boulfroy E. (2008). Les rôles de l'arbre en ville. Centre collégial de transfert de technologie en foresterie de Sainte-Foy (CERFO).

9. Mebirouk H. (2008). Entretenir, valoriser, et préserver les espaces verts dans les ensembles de logements collectifs à Annaba. Sciences \& Technologie D, (28), 31-38.

10. Merlin, P. et Choay, F. (1988). Dictionnaire de l'urbanisme et de l'aménagement. Paris ; PUF.

11. Nassa, D. D. A. (2009). Crise de la nature dans l'agglomération abidjanaise : l'exemple de la colonisation des espaces verts par l'habitat et les commerces dans la commune de Cocody. halshs-00352541. https://halshs.archives ouvertes.fr/halshs-00352541.

12. Polorigni, B., Radji, R. A. et Kokou, K. (2015). Politique publique de gestion des espaces verts de la ville de Lomé au Togo. International Journal of Biological an Chemical Sciences, 9(4), 1888-190.

13. Rolland, E. (2009). Villes et gestion des espaces verts : élaboration d'un outil d'évaluation qualitative. Essai de maîtrise et master, Université de Sherbrooke, Université de technologie de Troyes.

14. Tatar, S. et Nahal, S. (2016). Gestion des espaces verts en Algérie : Cas études de la ville de Tébessa (Quartier Copemad). Mémoire de fin d'études, Université Larbi Tébessi-Tébessa.

15. TRA, F. et IGBOU, G. N. (20017). Perceptions sociales et occupation des espaces verts à Gnanzoua dans la commune de Marcory (côte d'ivoire). Revue Africaine d'Anthropologie, Nyansa-Pô, (24), 194210. 